\title{
MUJER Y CINE EN AMÉRICA LATINA: PROYECTANDO UNA VISIÓN ALTERNATIVA DE LA NACIÓN
}

\section{Women and cinema in Latin America. An alternative vision of the nation}

\author{
Rosa-Linda Fregoso* \\ * Latin American and Latino Studies; University of California, Santa Cruz \\ rfregoso@ucsc.edu
}

Palabras clave

Cineastas latinas Interseccionalidad Nuevo cine latinoamericano

Keywords

Latina filmmakers Intersectionality New Latin American cinemas

\begin{abstract}
Resumen
Al explorar la relación entre cine de mujeres y cines latinoamericanos, este artículo interroga las categorías de cine nacional, cine latinoamericano y cine de mujeres con el fin de superar lógicas geopolíticas, de idiomas, colonialidad, y las lógicas industriales del mercado que han creado esas categorías. Partiendo de interrogantes como qué modelo de análisis feminista del cine es adecuado para articular las perspectivas de mujeres en las nuevas formaciones del cine mundial, propongo la utilidad del discurso de la interseccionalidad para un análisis de cine de mujeres que presente una visión alternativa de la nación.
\end{abstract}

\section{Abstract}

This exploration of the relation between women's cinema and Latin American cinemas, begins by interrogating the categories of national cinema, Latin American cinema, and women's cinemas, in order to transcend the geopolitical, linguistic, and colonialist and industrial market logics that have created these categories. Departing from questions such as: what feminist film analytics is adequate for articulating women's perspectives in new formations of world cinema?, I argue for the utility of the discourse of intersectionality as an analytics for films by women which present an alternative vision of the nation.

Fregoso, R-L. (2016). Mujer y cine en América Latina: proyectando una visión alternativa de la nación. Papeles del CEIC, vol. 2016/2, papel 161, CEIC (Centro de Estudios sobre la Identidad Colectiva), Universidad del País Vasco, http://dx.doi.org/10.1387/pceic.16330

En 1990, más de ochenta mujeres procedentes de México y los EE.UU. nos reunimos en la ciudad fronteriza de Tijuana, Baja California, en el primer "Encuentro de cineastas y videoastas mexicanas, chicanas, puertorriqueñas" titulado "Cruzando Fronteras". El encuentro fronterizo ocurrió durante la época que estaban de moda los encuentros de mujeres (o como solíamos decir, "los encuentros y desencuentros"), tres años después de aquel encuentro histórico "Cocina de mujeres" que se 
realizó en el Distrito Federal con la participación de un gran número de mujeres cineastas de varios países latinoamericanos y del Caribe.

Los encuentros de mujeres cineastas en esa época formaban parte de una larga trayectoria de actitud crítica y contestataria hacia los modelos clásicos de cine y los estereotipos de género, que surgió con la entrada de una nueva generación de realizadoras durante los años sesenta, que respondieron a las exigencias de su momento histórico - a las luchas colectivas de reivindicación, a los nuevos movimientos sociales de minorías en Estados Unidos (por la liberación de afroamericanos, chicanos y pueblos indígenas), movimientos revolucionarios de liberación nacional y descolonización, y por supuesto la lucha de liberación de mujeres-. Esa actitud contestataria de las cineastas de los años setenta en cada región dio paso a la formación de colectivos de cine-mujer para contrarrestar la realidad de marginalización de mujeres en las industrias nacionales de cinematografía, como el colectivo Cine Mujeres de México, que agrupó a feministas militantes de izquierda y otros grupos en Colombia y Venezuela.

El encuentro "Cruzando Fronteras" de 1990 coincidió con el auge de una nueva generación de realizadoras e investigadoras (la época del posmodernismo y multiculturalismo) que nos reunimos con el propósito de hacer conexiones con la cultura de cine de mujeres anterior, que se dedicó a traer a la pantalla la condición social de la mujer, a presentarnos una visión de mujer como sujeto activo y emancipado. Además, nos reunimos para intercambiar ideas y proyectos así como para rendir homenaje a pioneras del cine como Matilde Landeta, que con su película clásica La Negra Angustias, de 1949, expresó una incipiente visión contestataria [proto]-feminista, anti-racista y revolucionaria en México.

Cruzamos fronteras no solo conceptualmente, sino también físicamente, atravesando el puente entre Tijuana y San Diego para ver las más de ochenta películas y videos que se proyectaron durante el Encuentro. Fue un momento histórico en el que las categorías como "cine de mujer", la estética femenina, estética feminista, se estaban cuestionando, en parte por el movimiento intelectual anti-esencialista y posmodernista; fue un momento histórico en que las condiciones sociales y culturales del continente respondian a los cambios drásticos del neoliberalismo y pos-autoritarismo, y que las luchas colectivas de reivindicación especialmente en los EE.UU. habían entrado en una época 
de apogeo; fue un Encuentro en el cual debatimos: ¿qué es el cine de mujeres? y ¿qué es la mirada de mujer?

Veintiséis años después, he decidido retomar esa cuestión situándola en un marco más amplio, explorando la relación entre cine de mujeres y cines latino-americanos. ¿Qué modelo de análisis feminista del cine es adecuado para articular las perspectivas de mujeres en las nuevas formaciones del cine mundial? Lo que a primera vista parece una pregunta simple suele ser un problema teórico más complejo. Lo mismo se puede aplicar a la pregunta: ¿cómo entendemos el cine latinoamericano?

Cuando se refiere al "cine latinoamericano", se suele imaginar el cine procedente de América Latina, hecho por realizadoras de América Latina o quizás sobre latinoamérica. Algo parecido suele suceder con definiciones de cine nacional - argentino, uruguayo, mexicano- o sea, la definición está inscrita por un marco geopolítico, por el concepto de Estado-nación, como producción de una cineasta de un cierto país, interpretando relatos o temáticas particulares de la nación o la "cultura". En ciertos casos, la categoría de cine latinoamericano significa cine noHollywoodense, o no-europeo, o sea, cine extranjero al país de los que se encargan de crear ese señalamiento. Existe un problema similar con la categorización de cine mundial, la tendencia a definir la categoria en términos binarios, como cine del mundo, que se aleja del modelo hollywoodense, que es posicionado como la categoría normativa.

En otras palabras, el cine mundial suele definirse de forma "restrictiva y negativa, como cine que no es producido dentro del régimen de Hollywood" como Lucía Nagib (2006: 26) indica. Pero aparte de eso, las categorías geopolíticas resucitan problemas de binarismo y esencialismo cultural que no nos proporcionan un mejor entendimiento sobre la ecología compleja de cada filme agrupado dentro de las categorías nacionales/culturales y geopolíticas. Por eso, lo que ha escrito James Chapman en su libro Cinemas of the World, se puede aplicar al concepto cines latinoamericanos: "no está claro si es que algún modelo general pueda dar cuenta de las diversas prácticas cinematográficas, géneros, estilos y tradiciones que han surgido en el contexto global" (2003: 33).

El presente ensayo parte de la investigación que he realizado en las últimas tres décadas, enfocándome en poner de manifiesto las formas 
en que el poder opera en discursos y relaciones sociales, y en el caso del cine, las formas en que el poder opera para producir categorias que naturalizan fenómenos culturales. Me interesa comprender los límites de lo geopolítico y de lo "C"ultural (con mayúscula) como categorías organizadoras de cines latinoamericanos y el límite de conceptos como cine de mujer, porque esas categorizaciones no suelen explicar cómo las y los espectadores interpretan o ven los objetos estéticos ni cómo subjetividades y realidades son construidas por las formas creativas de expresión. Categorias que naturalizan fenómenos asumen una realidad a priori, que la realidad existe fuera de su construcción, y nos dicen muy poco sobre cómo un texto como el cine contribuye a figurar y a crear mundos.

Esta labor de descolonización de las categorias de cine nacional, cine latinoamericano, cine de mujeres para superar las lógicas geopolíticas, de idiomas y colonialidad, las lógicas industriales y del mercado que han creado las categorías, parte de un reciente proyecto de investigación sobre mujeres y cines en América Latina en el cual se me presentó la necesidad de buscar nuevas epistemologías que superen esas lógicas.

\section{EL NUEVO CINE LATINOAMERICANO: LA ONDA ANTERIOR}

Hace medio siglo, el movimiento que se conoce como el "nuevo cine latinoamericano" surgió en el contexto de las luchas revolucionarias en contra del neo-colonialismo, promoviendo la descolonización y liberación nacional, en su mayoría anti-capitalistas. Era un movimiento vanguardista con metas tanto políticas como estéticas, "con el fin de unir a Latinoamérica políticamente y culturalmente para confrontar el imperialismo y defender las identidades nacionales y regionales" (Villazana, 2013: 42). Este nuevo cine latinoamericano re-configuró y reimaginó nuevos espacios de representación para "comunicarse con el pueblo", pues los realizadores consideraban al sector popular como eje central del proyecto de concientización, lo cual declararon en una serie de manifiestos que enfatizaban una nueva política estética a favor del cine de guerrilla, tal y como lo expresaban Octavio Getino y Fernando Solanas en "Hacia un tercer cine" (1969), el brasileño Glauber Rocha, el boliviano Jorge Sanjinés y el cubano Julio García Espinoza en su formulación de cine imperfecto. 
El cineasta argentino Fernando Birri capta con precisión esas aspiraciones en su ensayo ahora canónico:
"el nuevo cine latinoamericano es un cine nacionalista, realista, crítico, y popular que trata de interpretar, expresar y comunicarse con el pueblo (...). Es un cine a favor de la liberación, de la liberación económica, política y cultural, y también la liberación de la imagen, es decir la imaginación" (citado en Fregoso, 1993: xx).

En ese sentido, el nuevo cine latinoamericano se proponía confrontar al "imperialismo cultural que consideraban como parte integrante del subdesarrollo" (Chanan, 2006: 40). Inspirados por el neorrealismo italiano (ya que varios cineastas estudiaron en el Centro Experimentale di Roma), buscaban crear un lenguaje cinematográfico nuevo, que testificara de una auténtica identidad nacional y que contribuyera a la liberación nacional del imperialismo cultural, de la hegemonía del modelo hollywoodense de colonizar la realidad y de las industrias cinematográficas de sus respectivas naciones.

Un eje central fue la búsqueda de "narrativas alternativas y diferentes lógicas representacionales que pudiesen fracturar" no solo la hegemonía del modelo hollywoodense, sino también el imaginario nacional de la élite burguesa. Los cineastas revolucionarios criticaban la producción de cine nacional, particularmente el género de melodrama, que atribuyeron a una imposición colonialista, un imperialismo cultural procedente de Hollywood (Shaw y Dennison, 2005: 4). Oponiéndose al cine comercial de la industria nacional y la producción de cine de entretenimiento y distracción, el movimiento del nuevo cine latinoamericano contrarrestó el supuesto imaginario de la nación, dándole voz a sujetos marginados o subalternos que han sido sistemáticamente excluidos de la definición de la nación, el Estado, la ciudadanía, y por medio de eso desatando su potencial revolucionario.

Si los realizadores del nuevo cine visualizaban América Latina como una "comunidad de países que compartían un mismo destino", un "pluralismo radical", o como Fernando Birri dirá "el uno en lo diverso"-lo que en el siglo XIX José Martí nombraría "Nuestra América"-, esta formulación de un hemisferio integrado formó también parte de las estrategias comerciales de los Estados Unidos que en la década de los 40 con el programa "Good Neighbor Policy" (Administración de Roosevelt) 
consideró América Latina como un mercado singular para los distribuidores de películas de Hollywood (Alvaray, 2008).

En la época neoliberal, "el uno en lo diverso" tiene otro significado. Según Luisela Alvaray, "en la noción adoptada por los cineastas del nuevo cine latinoamericano de un continente integrado, la unidad formaba parte de una conciencia política, de una necesidad de documentar las injusticia y desigualdades que afligian a los países latinoamericanos" (2008: 57). Sin embargo, en la época neoliberal, "el uno en el diverso", se ha transformado en una lógica del mercado.

Las transformaciones políticas y económicas a lo largo de la región han tenido mayores impactos en las industrias de mayor producción cinematográfica. El fin de las dictaduras militares de los setenta y el giro democrático han abierto nuevos espacios políticos a la vez que se han transformado las industrias cinematográficas por medio de nuevas estructuras de financiamiento a través de una lógica neoliberal de mercado libre. Como resultado de la crisis económica que afectó a varios países en la región, los gobiernos adoptaron políticas de austeridad y la retirada de financiamiento estatal en países como Brasil, Argentina y México.

Según Chanan, "en Argentina, la producción disminuyó dramáticamente de 46 filmes en 1982 a 4 en 1989. Para ese mismo año, en Brasil, la producción disminuyó de cientos de películas los años anteriores a 20 en 1989" (2006: 44). La devaluación del peso en México (el denominado "efecto Tequila") y el Tratado de Libre Comercio tuvieron un efecto similar no solo en la producción, sino en la exhibición de películas mexicanas, ya que como nos dice la cineasta, María Novaro, "veinte años después no hemos logrado reestablecer algo sano de acceso a nuestras propias pantallas" (2015).

A la vez, representantes oficiales de políticas de cine han adoptado y promovido el objetivo de un "continente integrado" en América Latina, como nos indica Alvaray en su investigación sobre "las nuevas ondas del cine latinoamericano". Según Alvaray:

\footnotetext{
"sin perder de vista la importancia de las películas para la determinación cultural y nacional, representantes de los gobiernos han firmado varios acuerdos oficiales autorizando un mercado común de cine y la promoción de coproducciones. En 1984, representantes gubernamentales de España, Portugal, y diez países latinoamericanos se
} 
reunieron en Madrid para formular estrategias con las que aprovechar el mercado Iberoamericano, que ya que contaba con 1.2 billones de espectadores y era potencialmente un mercado viable y rentable para coproducciones en portugués y español" (2008: 57-58).

Esta estrategia transcontinental de coproducciones y distribución conservaba la apariencia de la lucha contra el imperialismo cultural, en los esfuerzos de contrarrestar la hegemonía del régimen (máquina) de Hollywood.

En su libro sobre cine en América Latina, Octavio Getino escribe:

\begin{abstract}
"Las tentativas de integración regional del cine 'latinoamericano' y por extensión del 'iberoamericano', estuvieron presentes en numerosas declaraciones y acuerdos suscriptos entre nuestros países a lo largo de más de seis décadas. Finalmente, comenzaron a tener fuerza de ley (...) cuando se firmaron en la ciudad de Caracas, en octubre de 1989, los primeros documentos formales sobre la integración, coproducción y Mercado común, que fueron ratificados por los congresos nacionales de más de una decena de países de Iberoamérica" (1998: 13).
\end{abstract}

Instituciones tanto como lbermedia y Mercosur y festivales internacionales de cine dedicados al cine latinoamericano e iberoamericano han fomentado la promoción, distribución y exhibición del cine latinoamericano (Ibermedia fue creada en 1997 con 14 paísesmiembros). Como indica Alvaray, el Mercado Cinematográfico de Mercosur, creado en 2005, ha desempeñado un rol fundamental en "coproducciones" y en el desarrollo de "la idea de una América Latina unida", en donde "espacios (como el mercado y el festival)" servirán como plataformas para el lanzamiento de películas latinoamericanas en las pantallas mundiales" (2008: 59).

Anteriormente señalamos que durante la primera etapa del nuevo cine latinoamericano el proyecto principal era unir a América Latina culturalmente y políticamente con el fin de enfrentar al imperialismo cultural y defender las identidades nacionales. En el nuevo contexto neoliberal de producción y distribución de cine, ¿cómo se puede conceptualizar el cine latinoamericano? ¿Cómo suman todos los cines nacionales en América Latina? Ciertamente, las nuevas lógicas del mercado que animan "las políticas nacionales, acuerdos regionales, estrategias de marketing" han dado forma a la manera cómo 
entendemos los cines latinoamericanos, como formación que transciende el "marco parcial del Estado-nación o las particularidades de lo local".

Lo que queda claro es el impacto de la globalización neoliberal en la transformación del modelo centro/periferia asociado con el Estadonación ya que los nuevos contextos de producción y distribución transcienden espacios nacionales, dado que flujos globales y contextos de intercambio transnacional siguen acelerando las interconexiones de gente por los medios de comunicación, la migración, el capitalismo global. Una perspectiva globalizada sobre cine latinoamericano como categoría general con especificidades locales/nacionales es la base de una nueva lógica de mercado del cine latinoamericano -una formulación neoliberal sobre la diversidad cultural que re-significa la fórmula de Birri, "el uno en el diverso" y, como mencioné anteriormente, tiende a desenterrar problemas de binarismo y esencialismo cultural-.

\section{CUANDO UNA MUJER MANEJA LA CÁMARA}

El Encuentro "Cruzando Fronteras" se llevó a cabo en la coyuntura de estos eventos: de los ajustes estructurales y transformaciones, o sea, la re-formación de la industria cinematográfica en varios países a raíz de políticas económicas y culturales. Nos reunimos como una ONG, pero sin patrocinio de instancias transatlánticas o inter-regionales, para criticar la marginación de realizadoras no solo en la historia que se narra sobre el movimiento del nuevo cine latinoamericano, sino en las industrias de cine nacional. Escuchamos anécdotas sobre esta marginación como la de Maria Novaro "en el 1985, me topé con el horror que en los estatutos del sindicato cinematográfico decía que 'las mujeres no podían tocar la cámara'" (2015).

Durante los cuatro días que pasamos juntas en el Encuentro intercambiamos ideas sobre la inscripción textual del género, sobre la imagen de la mujer como óptica para cuestionar la construcción de la nación y visibilizar una sensibilidad estética subversiva. Fue la primera vez en que realizadoras chicanas y puertorriqueñas se sentaron en la mesa con mexicanas para escucharnos hablar sobre el rol del género y la sexualidad en obras audiovisuales hechas por las mismas realizadoras. Intercambiamos perspectivas sobre esa cuestión de enorme calado: ¿existe o puede existir una estética femenina? Registramos cambios en 
el discurso femenino audiovisual, la estética femenina como un movimiento de lo exterior al interior, de lo público a lo privado, lo que Ruby Rich llamó entonces un cambio de lo revolucionario a lo revelador, y Ana Marta Mier denominó como el movimiento hacia la individualidad y el abandono de la colectividad (Iglesias y Fregoso, 1998).

Fue en ese primer encuentro que cineastas, videoastas y académicas cruzamos fronteras para intercambiar ideas sobre cómo se inscribe el género en el texto audiovisual, sobre la relación entre protagonismo (autoría) y estética (representación), sobre la mirada e imagen de la mujer como óptica para cuestionar la construcción imaginaria de la nación, sobre el género como óptica además para visibilizar una sensibilidad estética subversiva.

Como aprendimos de la generación anterior de cineastas que participaron, los escenarios sociales y culturales que enfrentábamos en los años ochenta y noventa eran mucho menos adversos. Fue por eso que pudimos formular ideas como la que presentó Marisa Sistach de la perspectiva de la mujer desde la "esfera de la intimidad", porque es un "lenguaje que nos jala", que comunica otro punto de vista, otra sensibilidad, otra subjetividad" (Iglesias y Fregoso, 1998, 271).

Margara Millán hizo un llamado para elaborar "desde la especificidad de la diferencia, una estética cinematográfica", que sea lejos de "un programa o un canon a seguir, (...) un proceso liberador que pasa por la auto-conciencia de las elecciones" - una estética de la responsabilidad creadora, ni restrictiva ni intolerante, solo consciente de los signos que emplea, de su relación con una estructura precisa, de su selección" (1998: 126).

Fue allí, en ese espacio híbrido y cruzado, donde formulamos una conciencia de la diferencia y una noción de liberación a través de la diferencia. Sin llegar a un acuerdo sobre cómo se define "cine de mujeres" o "mirada de mujeres" (que además fue el título del libro que publicamos sobre el encuentro), lo que quedó sumamente claro es que hay otras maneras de organizar el mundo cinematográfico que tomen en cuenta otras identidades que superan el "Estado-nación", que en "un sistema de relaciones, contradicciones, desfases, cambios y continuidades", como nos explica Patricia Torres, las realizadoras de imágenes audiovisuales "se han abierto brecha", y que esa responsabilidad creadora de la que nos hablaba Millán representaba una 
vinculación de relatos, estilos y temáticas con discursos transgresores, dirigidos en gran medida a un público crítico y exigente" (Torres, 2004: 9).

No pudimos llegar a un acuerdo sobre la categoría de cine de mujeres, animadas por la interrogación: ¿Cómo entonces, se define la categoría "cine de mujeres"? ¿por su autoría, como cine hecho por mujeres? ¿o por contenido, como cine sobre mujeres? ¿cómo cine que captura una sensibilidad femenina? ¿o por I

a reivindicación feminista, como cine hecho por y para mujeres? Fueron preguntas que no resolvimos entonces y que veintiséis años después me siguen persiguiendo. Aunque fue en ese Encuentro histórico de 1990 donde hicimos un primer intento de articular un imaginario feminista transfronterizo o transnacional, con el fin de rebasar las limitaciones conceptuales del Estado-nación y de re-pensar la relación entre género y cine desde un marco transnacional con el propósito de examinar las relaciones de poder y conocimiento entre y dentro de culturas, un momento en que articulamos distintas formas de descolonizar la mirada.

"Cruzando Fronteras" abrió un espacio conceptual para mí a través del contexto transnacional de intercambio e intersubjetividad. Aquel encuentro histórico afirmo la idea y la importancia de aproximarse a los textos desde un análisis discursivo tomando en cuenta a la vez la multiplicidad de estilos y perspectivas femeninas y feministas, en vez de tratar de aplicar un eje singular o una definición restrictiva o esencialista del cine de mujeres.

Este intercambio transnacional de latinas cruzando fronteras fue un instante de enredo, de cruces-culturales. Para dar un ejemplo, la especificidad de la diferencia (el llamado de Millán), o mejor dicho, cómo se conceptualiza la "diferencia" fue una de las "diferencias" entre las Latinas del norte de la frontera y las del sur. Las que nos ubicamos en los EE.UU., como Frances Negrón Muntaner, Carmen Huaco-Nuzum, Lilian Jiménez, entre otras, trabajábamos el discurso feminista de mujeres de color (women of color feminism) que en la década de los noventa inspiró al "feminismo transnacional".

El feminismo de mujeres de color elaborado por feministas afroamericanas (Patricia Hill Collins, Angela Davis, bell hooks, Kimberlé Crenshaw), chicanas/latinas (Cherrie Moraga y Gloria Anzaldúa), y el 
colectivo Combahee River, consideraba la "especificidad de la diferencia" con la óptica de la interseccionalidad, una óptica que considera la arquitectura de género no solo en términos de las relaciones entre mujeres y hombres, sino en términos de las relaciones sociales de clase, razas/etnias y sexualidades. Fue esta interacción de diferencias de clase, raza, género y sexualidad que para las latinas del norte de la frontera, en ese momento histórico, representó una estrategia fundamental para cualquier elaboración de una estética, porque la interseccionalidad estructura epistemologías alternativas y un cuestionamiento de la modernidad, elementos que forman las bases de un modelo intervencionista o practica estética insurgente.

Aunque en muchos casos el discurso de la interseccionalidad apunta a identidades interconectadas, por ejemplo género/sexo, raza/etnia, clase, religión, etc., el discurso de la interseccionalidad es fundamentalmente un método para pensar más allá de análisis basados en un eje singular, el más allá del modelo binario de conceptualizar la raza como separada de género, como separada de clase, de sexualidad, etc., y de emprender la investigación sobre las estructuras que impacten en las vidas de mujeres subalternas. En otras palabras, el discurso de la interseccionalidad rechaza la desarticulación o el hecho de desconectar los regímenes de poder que estructuran la subordinación de mujeres, como el racismo, el patriarcado, el capitalismo, la heteronormatividad.

En el campo político actual, el discurso feminista de la interseccionalidad elaborado por feministas negras propone la importancia de la interconexión entre luchas en contra de la violencia de género con luchas en contra de la violencia de estado, de violencia sexista institucional y violencia sexista cotidiana que sufren las mujeres.

Sigue siendo productivo el discurso de la interseccionalidad, ya que hasta la fecha lo sigo aplicando para el análisis de películas hechas por mujeres, como en el caso de Madeinusa (2006), la controvertida película de la cineasta peruana Claudia Llosa, a quien se le acusó de reproducir estereotipos racistas sobre el pueblo andino en el Perú. El relato se lleva a cabo en una comunidad ficticia en los Andes que cada año celebra el "tiempo santo" - tres días de fiesta, de Viernes Santo a Domingo de Resurrección-cuando para ese pueblo ficticio quechua hablante, "Dios no existe", o sea el Cristo es vendado y simbólicamente está muerto. 
Durante los tres dias de Tiempo Santo, las normas de conducta son suspendidas y no puede haber pecado, ya que nada es prohibido, convirtiendo lo más sagrado en lo más profano. En un medio ambiente carnavalesco toda forma de exceso, desorden y libertinaje puede lograrse ya que como Dios está muerto, no puede observar y por consiguiente castigar los pecados de la carne.

Un evento culminante durante las fiestas es la selección y coronación de una jovencita como "La Virgen de Manyaycuna", que la comunidad elige entre las "vírgenes" jóvenes del pueblo, la visten de Virgen María y después de una procesión, se la entregan al alcalde que tiene relaciones sexuales con ella, o sea, que se encarga de violar a la jovencita.

La película empieza con la llegada inesperada de Salvador, un criollo de Lima, que llega en autobús y se ve obligado a permanecer en el pueblo hasta que pase la tormenta. Ya que la llegada de Salvador coincide con el Tiempo Santo, el ayuntamiento del pueblo decide mantenerlo en cautiverio en casa del Alcalde Don Chayo. Y es allí donde Salvador conoce a Madeinusa, la hija del Alcalde, que ha sido elegida como la Virgen de Manayaycuna.

Al paso de la elaboración del relato nos enteramos que lejos de ser un honor para Madeinusa, le repugna la idea de asumir el rol de Virgen y que lo que más desea es encontrar a su madre que huyó del pueblo bajo circunstancias misteriosas. Cuando es testigo de la violación incestuosa de Madeinusa por el Alcalde Don Chayo (su padre), Salvador se compromete a ayudarla a escapar. Antes de partir, Madeinusa regresa a casa a recoger los aretes de su madre y al descubrir que su padre los ha destruido, decide vengarse y envenenarlo con veneno de rata que le pone al caldo de pollo. Cuando Salvador llega y encuentra a Don Chayo en espasmos, se lo reprocha a Madeinusa y tanto ella como la hermana responden gritando, "el gringo mató al Alcalde". En la última escena vemos a Madeinusa viajando sola a Lima, lo cual nos indica que seguramente Salvador enfrentó a la justicia comunitaria por asesinar a Don Chayo.

A pesar de su éxito internacional, dentro del país la película recibió reseñas muy negativas. La inteligencia peruana condenó a Llosa por distorsionar al pueblo indígena del Perú, por reproducir estereotipos racistas del pueblo andino, por exotizar y menospreciar al pueblo quechua-hablante, y por su fabricación de festividades y rituales como 
el "Tiempo Santo". A Llosa se le acusó de incitar a los prejuicios que los criollos tienen sobre el pueblo andino y de reproducir el discurso colonialista binario de civilización y barbarie que históricamente prevalece y sustenta no solo en el imaginario nacional sino del continente americano (Roca, 2006).

No pienso negar que la película Madeinusa anima o alienta una mirada colonialista, ya que siglos de estereotipos colonialistas de los pueblos originarios han sido representados como bárbaros, pre-modernos, impregnados de tradiciones y rituales primitivas y en gran medida la cineasta Claudia Llosa participa en esa larga trayectoria histórica -lo cual plantea cuestiones políticas, ideológicas e históricas para los que trabajamos dentro del marco de una praxis decolonialista-. Sin embargo, a pesar de que la película es contradictoria en su tratamiento de las prácticas indígenas comunales, hay que reconocer el significado de su crítica a las relaciones de poder patriarcales dentro de los pueblos originarios. Y la película logra esa crítica cuando empodera la subjetividad de una mujer indígena, enfocando su mirada en el deseo de Madeinusa de libertad por encima de todos los demás deseos (amorosos, reina/virgen del pueblo). A pesar de sus buenas intenciones, las críticas tienden a des-empoderar retóricamente a los sujetos que pretenden defender o por quienes abogan, como el caso de Madeinusa. Inspirada por el argumento de Gayatri Spivak en su célebre artículo, "Can the Subaltern Speak?" (1988), propongo la necesidad de una crítica cultural con mayores matices, para eludir el problema de retóricamente des-empoderar al sujeto subalterno femenino y quitarle la voz.

El deseo de la protagonista de liberación depende del abandono de la autoridad religiosa, secular y liberal, "cada una representada por tres figuras masculinas que mueren durante el desarrollo de la trama: Jesucristo, Don Chayo y Salvador" (Beasley-Murray, 2008). En este sentido, lo que la crítica negativa de la película ignora es precisamente la interseccionalidad de regímenes de poder colonialista - el sistema racista y patriarcal- en la producción de violencia en contra de mujeres indigenas.

Claudia Llosa representa las violencias corporales y epistémicas a través de la lente de la interseccionalidad, mostrándonos cómo las estructuras de dominación, arraigadas en la colonialidad del poder, son también el fundamento del des-empoderamiento de los sujetos coloniales femeninos. Los críticos de Madeinusa fallan en reconocer el gran 
significado de representar el deseo de una joven indigena ligada a la destrucción de las instituciones entrelazadas que han causado la abyección de y daños a las mujeres de los pueblos originarios y de esa forma no reconocen la importancia de la crítica a la colonialidad de poder alegorizada por la película.

Es necesario articular una crítica cultural que convalide y valore la posición del sujeto femenino indígena, que afirme el deseo por la liberación y el empoderamiento; una perspectiva que haga visible los grilletes que obstruyen la liberación y libertad de Madeinusa. Claudia Llosa representa estos grilletes en términos de los siguientes regímenes de poder arraigados en la colonialidad:

1. La religión: la semana santa, una festividad impuesta por la iglesia católica europea durante la colonización, incluyendo el culto de Virgen conocido como "Marianismo".

2. Gobernación: el alcalde del pueblo manda por medio de la jerarquía colonialista en vez de por formas de gobernación andinas, pues hasta la fecha el Ayllu es parte de los usos y costumbres de democracia comunal practicadas por los pueblos Aymara y Quechua de los Andes.

3. Violencia sexual: el derecho de pernada, conocido también como prima note, es una forma de violencia sexual patriarcal que fue introducida durante la conquista y practicada por latifundistas, cuyos vestigios permanecen hasta la fecha y que en su forma originaria implica un ejercicio de derecho sexual por parte del amo sobre la hija del vasallo. La violación colonialista codificada en el "derecho de pernada" también se ha perpetuado por parte de latifundistas que lo ejercen contra hijas de campesinos. En la película el Alcalde/Padre de Madeinusa ocupa el rol simbólico del latifundista que ejerce su derecho de pernada contra su hija.

Como hemos notado, la libertad y liberación de Madeinusa solo se puede lograr a través de la destrucción de los regímenes colonialistas de género y raza que han sido incorporados por formas de gobernanza, prácticas religiosas y relaciones sociales. Estos regímenes de poder colonialista son alegorizados en las tres figuras protagonistas en la película: Jesucristo/la autoridad católica de la conquista; el Alcalde/padre, representante del patriarcado y el estado colonialista; y Salvador, el criollo/salvador blanco de Lima. 
La selección de "Salvador" como el nombre del criollo blanco es un gesto feminista brillante para contrarrestar el estereotipo de que las mujeres subalternas o en este caso indigenas necesitan de un salvador blanco que las rescate de la barbarie/salvajada de los hombres indigenas ${ }^{1}$. La muerte discursiva y literal de estas figuras alegóricas que representan estructuras de poder colonialista abre un espacio para la subjetividad agencial (agential subjectivity) de Madeinusa y un camino hacia su libertad y liberación. El discurso de la interseccionalidad que han elaborado las feministas de color posibilita un entendimiento más matizado sobre la disonancia, contradicción y ambivalencia que informa la perspectiva de Claudia Llosa sobre la indigeneidad y a la vez contribuye a una crítica cultural feminista acerca de la interseccionalidad de estructuras de dominación que causan daño a la vida de una mujer indígena.

\section{CODA}

Patricia White nos sugiere la importancia de ubicar y localizar históricamente a la realizadora, la investigadora, el texto y las prácticas de recepción: una orientación feminista posiciona a las películas realizadas por mujeres en relación a discursos de agencia (autoría) como a los de estética (representación) (White, 2015: 15). De ese pensamiento parten mis reflexiones sobre el encuentro "Cruzando Fronteras" y su impacto en $\mathrm{mi}$ pensamiento feminista sobre la producción cinematográfica de mujeres.

En la investigación, el énfasis es en el cine de mujeres que responde tanto a espectadores como a preocupaciones locales y transnacionales, $y$ en el cine de mujeres como una forma alternativa de imaginar la nación. Esta forma alternativa de imaginar la nación implica una crítica a la modernidad como proyecto colonialista y universal que impone jerarquías sociales racistas, patriarcales y hetero-normativas, que homogeniza las diferencias culturales bajo un sujeto nacional $y$ masculino, supuestamente homogéneo, o que representa una unidad y coherencia nacional. Mi interés es en trabajos que presenten nuevas estrategias productivas y miradas frescas sobre la (in)coherencia de seres culturales, raciales, sexuales, en las periferias y fronteras

\footnotetext{
${ }^{1}$ Este argumento es elaborado por Beasley-Murray en su excelente artículo (2008). 
colonialistas; en propuestas fílmicas que transgredan las identidades colonialistas de género, rígidamente definidas.

En este sentido hay menos interés en reproducir categorías como "cine de mujer", ya que como nos advierten Parvati Nair y Julián Daniel Gutiérrez-Albilla: "nos preguntamos si podría ser visto como una categoría en sí misma el cine hecho por mujeres ¿se puede decir que existe tal categoría antes de la intervención de académicos? ¿o es de hecho un producto de la Academia, de estudios feministas de cine y de teóricos de género?" (Nair y Gutiérrez-Arbila, 2013: 7) El auge de la nueva generación de realizadoras de cine en América Latina coincidió con el cuestionamiento de categorias culturales esencialistas de identidades sociales como la de género, y con la afirmación de la pluralidad y la alteridad de perspectivas. $y$ es precisamente esta diversidad y heterogeneidad en prácticas cinematográficas la que dificulta la idea de categorías singulares o "el espacio de 'cine de mujeres"' (Nair y Gutiérrez-Arbila, 2013: 8).

Obviamente estoy de acuerdo con las posturas de las teorías formuladas por el imaginario transnacional feminista sobre estudios de cine que proponen que la crítica feminista sobre el cine debe transcender "el marco restrictivo del Estado-nación y resituar críticamente al género y al cine en un marco transnacional que posibilite o haga posible la examinación de relaciones de poder y conocimiento entre y dentro de culturas", como nos dice Ligzhen Wang (2011: 2). Para el contexto sociohistórico de Latinas de las Américas, es necesario elaborar modelos de análisis de cinema que recurran a discursos como el de la descolonialidad e interseccionalidad.

Al mismo tiempo hay que reconocer "la interconexión de prácticas cinematográficas que transcienden/al más allá de las fronteras nacionales" (Dennson y Lim, 2006: 10). Porque "los espacios dentro de las fronteras nacionales", como sugieren Dennison y Lim, "ya están híbridos y cruzados, lo cual no disminuye o socava la necesidad de seguir cruzando todo tipo de fronteras, incluyendo nacional, políticas, de género" raza, sexualidad (Dennson y Lim, 2006: 10).

\section{BIBLIOGRAFÍA}

Alvaray, L. (2008). National, Regional, and Global: New Waves of Latin American Cinema. Cinema Journal, 47(3) (Spring), 48-65. 
Beasley-Murray, J. (2008). Subalternidad, Traición y Fuga. In L. DunoGottberg (Ed.), Miradas al margen y subalternidad en el cine latinoamericano y del caribe (pp. 367-392). Caracas: Fundación Cinemateca Nacional.

Chanan, M. (2006). Latin American cinema: from underdevelopment to postmodernism. In S. Dennison \& S. H. Lim (Eds.), Remapping World Cinema: Identity, Culture and Politics in Film (pp. 38-51). London: Wallflower Press.

Chapman, J. (2003). Cinemas of the World: Film and Society from 1895 to the Present. London: Reaktion.

Dennison, S. \& Lim, S. H. (2006). Introduction. In Remapping World Cinema: Identity, Culture and Politics in Film (pp. 1-15). London: Wallflower Press.

Fregoso, R-L. (1993). The Bronze Screen: Chicana and Chicano Film Culture. Minneapolis: University of Minnesota Press.

Getino, O. (1998). Cine y televisión en América Latina: Producción y mercados. Santiago/Buenos Aires: LOM Ediciones, Ediciones CICCUS.

Getino, O. \& F. Solanas (1969) "Hacia un tercer cine," Tricontinental, 14 (Octubre), 107-132.

Iglesias, N. \& Fregoso, R-L. (Eds.). (1998). Mirada de Mujer, Tijuana y Berkeley: Colegio de La Frontera Norte y Chicana/Latina Research Center.

Millán, M. (1998). Mirada de Mujer. In N. Iglesias \& R-L. Fregoso (Eds.), Mirada de Mujer (pp. 121-126), Tijuana y Berkeley: Colegio de La Frontera Norte y Chicana/Latina Research Center.

Nagib, L. (2006). Towards a positive definition of world cinema. In Remapping World Cinema: Identity, Culture and Politics in Film (pp. 26-33). London: Wallflower Press.

Nair, P. \& Gutiérrez-Albilla, J. D. (Eds.). (2013). Introduction. In Hispanic and Lusophone Women Filmmakers: Theory, Practice and Differences. Manchester: Manchester University Press.

Novaro, M. (2015). Academy Visual History Project. Entrevista realizada por Lourdes Portillo, 25 de septiembre de 2015.

Roca, P. (2006). Madeinusa o el insulto hecho cine. Centro de Medios Independientes Perú, 25 de septiembre.

Shaw, L. \& Dennison, S. (Eds.). (2005). Latin American Cinema: Essays on Modernity, Gender and National Identity. Jefferson, North Carolina: McFarland Inc. 
Spivak, G. Ch. (1988). Can the Subaltern Speak? In C. Nelson \& L. Grossberg (Eds.), Marxism and the Interpretation of Culture (pp. 271-313). Urbana: University of Illinois Press.

Torres, P. (2004). Mujeres y cine en América Latina. Guadalajara: Editorial Universidad de Guadalajara.

Villazana, L. (2013). Redefining Transnational Cinema. In S. Dennison (Ed.), Contemporary Hispanic Cinema (pp. 25-41). Woodridge: Tamesis.

Wang, L. (Ed.). (2011). Introduction. In Chinese Women Cinema: Transnational Contexts. New York: Columbia University Press.

White, P. (2015). Women's Cinema, World Cinema. Durham: Duke University Press. 